\title{
ESTUDIOS
}

\section{El fraude en la información empresarial en el marco de las relaciones financieras'}

\section{Horacio D. Molina Sánchez²}

Resumen: El sistema financiero se fundamenta en la confianza. La información es la base sobre la cual se toman las decisiones y su veracidad es clave para mantener la confianza. El fraude en la información altera seriamente este pilar del sistema financiero y genera alarma social. El artículo presenta la metodología de Cressey para la detección del fraude y que conceptualiza que en todo fraude se dan cita una presión o incentivo en la comisión de un fraude; una oportunidad de poder cometerlo y no ser detectado y una racionalización por parte del defraudador que justifique éticamente su comportamiento. Sobre la base de estos tres pilares el artículo revisa diversos debates que tratan de mitigar las conductas fraudulentas.

Palabras clave: Fraude, Gobierno Corporativo, Auditoría.

Fecha de recepción: 23 de febrero de 2015.

\footnotetext{
' El tema del fraude y la corrupción es muy amplio, por ejemplo, el fraude fiscal, el fraude laboral, la apropiación indebida o el fraude en la información empresarial en el marco de las relaciones financieras. En este trabajo nos referimos a este último, si bien muchas de las conclusiones que se puedan extraer son válidas para otras modalidades de corruptelas.

2 Profesor titular de área de la Universidad Loyola Andalucía. Deseo expresar mi agradecimiento a los profesores Antonio Barral, Rafael Bautista y Jesús N. Ramírez, profesores de la Universidad Loyola Andalucía, por sus valiosos comentarios a una primera versión de este texto.
} 


\section{Fraud in business information in the context of financial relations}

\begin{abstract}
Financial system is based on confidence. Information is placed at the roots of decision taking and its fairness is critical for maintaining the confidence. Information's Fraud seriously alters this pillar of financial system and creates social alarm. This paper shows Cressey's methodology for detecting fraud and conceptualizes that in any fraud meets a pressure or incentive for committing the fraud, an opportunity for doing it and avoiding its discovery and a fraudster's reasoning that ethically justifies his/her behavior. On these bases, the paper reviews several debates that deal to mitigate fraudulent behaviors.
\end{abstract}

Key words: Fraud, Corporate Governance,Audit.

\section{Fraude dans les informations de l'entreprise dans le contexte des relations financières}

Résumé: Le système financier repose sur la confiance. L'information est la base sur laquelle les décisions sont prises et leur véracité estessentielle pour maintenir la confiance. Fraude dans l'information sérieusementaltére ce pilier du système financier et génére une alarme sociale. L'article présente une méthodologie de Cressey pour détecter les frauds. II a conceptualisé que dans toute fraude existe pression ou incitation à commettre une fraude; une occasion de le faire et ne pas être détecté et la rationalisation par le fraudeur sur le plan éthique qui justifient son comportement. Sur la base de ces trois piliers l'article passe en revue différents débats qui cherchent à atténuer les comportements frauduleux.

Mots clé: Fraude, gouvernance d'entreprise, audit.

\section{Introducción}

La confianza es uno de los pilares del funcionamiento del sistema financiero. Sin confianza los acreedores no prestarían fondos a las entidades o los inversores no situarían sus recursos en las empresas. El incremento de la confianza mejora la asignación de recursos en el sistema financiero.

Estos aportantes de fondos (prestamistas e inversores entre los más significativos) toman sus decisiones en base a la información que preparan, en su origen, las entidades receptoras de los fondos y confían la gestión a unos directivos a los cuales posteriormente evalúan en función de la información que les suministran. No es preciso ser muy perspicaz para caer en la cuenta que los aportantes de recursos necesitan dotarse de instrumentos eficaces para evitar 0 , al menos, mitigar las amenazas que minan la confianza que necesitan en sus procesos de decisión. La academia viene estudiando estos problemas desde hace tiempo. 
Por un lado, la asimetría de la información que afecta entre otros mercados a los financieros y el estudio de las consecuencias que de ella se derivan fueron objeto del reconocimiento del premio Nobel de Economía a Akerloff, Spence y Stiglitz en 2001. Las diferencias de información entre el preparador de la misma y su usuario provocan que el segundo pueda realizar una selección adversa, guiándose por indicadores como el precio, cuando la calidad ha sido ocultada, o que incurra en un elevado riesgo moral que consiste en una conducta desleal del receptor de los fondos que los asignaría a proyectos de mayor riesgo que el inicialmente evaluado para la aportación.

Por otro, el estudio de los problemas de agencia que explican las relaciones entre los directivos de una entidad (agentes) y los propietarios (principales) ha sido uno de los campos donde se han estudiado los problemas de asimetría en la información entre las partes contratantes. La relación de agencia se fundamenta en la información y ésta es preparada por los agentes.

Para paliar los problemas relacionados con la relación de agencia se instituyen ciertos mecanismos, unos destinados a a linear los intereses del agente con los del principal y otros destinados a evitar los problemas de riesgo moral. Entre los mecanismos más institucionalizados están la auditoría externa de los estados financieros, numerosos pasajes de la legislación mercantil en defensa de los intereses de las minorías, los que promueven prácticas de buen gobierno y de control interno eficaces en las entidades o, finalmente, los órganos de supervisión de los mercados de valores y financieros destinados a garantizar el acceso en igualdad de condiciones a una información de calidad. Además de estas instituciones jurídicas, existen prácticas destinadas a reducir los costes de la relación de agencia, como por ejemplo, las prácticas de retribución en función del desempeño a los directivos.

En ocasiones, los mecanismos producen efectos contrapuestos; así, por ejemplo, una política de retribución a los directivos basada en la creación de valor para la entidad alinearía los intereses de los propietarios con los de sus directivos. Sin embargo, esta forma de retribución puede favorecer el cortoplacismo en las decisiones de los directivos y es un terreno abonado para el empleo de políticas contables y de gestión agresivas, cuando no fraudulentas, para tratar de cumplir esos objetivos. Aunque los paquetes retributivos se suelen diseñar con carácter plurianual, cuando llega el momento de la consolidación de los derechos por parte de los directivos o cuando perciben su retribución, normalmente en acciones, el riesgo de maquillaje de cifras se eleva.

La manipulación de la información supone una quiebra de la relación de confianza básica que requieren las instituciones del sistema financiero para funcionar efi- 
cientemente. Conforme aumenta el riesgo de manipulación de las cifras también se incrementan los costes de la relación de agencia. Esta ha sido una tendencia que venimos observando en los últimos tiempos, con un progresivo incremento de la regulación tendente al control de riesgos asumidos por los directivos, el reforzamiento del control sobre las firmas de auditoría externa y un mayor nivel de regulación en las prácticas destinadas a regular la relación de agencia, como la retribución a los directivos.

La literatura señala dos tipos de fraude: el fraude por apropiación y el fraude en la información (International Auditing Standard, 240 The auditor's responsibility relating to fraud in an audit of financial statements; Center for Audit Quality $\left.{ }^{3}, 2010\right)$. Ambos están estrechamente interrelacionados; el fraude por apropiación suele ir unido a uno en la información para ocultar la actividad fraudulenta, mientras que el fraude en la información, en un gran número de ocasiones se vincula a uno de apropiación, bien por la consecución de un incentivo ilegítimamente, bien por evitar un despido.

Las cifras del fraude son significativas. Uno de los estudios mejor documentados es el de Beasley et al (2010), auspiciado por el Committee of Sponsoring Organizations of the Treadway Commission (COSO). Este estudio analizó las acciones emprendidas por la SEC por fraude en la información financiera, en el periodo 1998 a 2007, donde detectaron 347 casos (que contrastan con los 294 casos del decenio anterior, 1987 a 1997). El valor total de los fraudes identificados en dicho estudio supera los 120 billones de dólares, presentando unos pocos casos con unos valores muy elevados (por ejemplo, Enron o Worldcom) y en un $89 \%$ de los casos se observó algún tipo de involucración por parte de los directivos $(83 \%$ en el decenio anterior).

La academia y el mundo profesional han elaborado una amplia literatura al respecto, existiendo revistas académicas especializadas, certificaciones profesionales y todo un campo de actuación, que se denomina "forensic accounting" y dentro de éste uno específico denominado "fraud examination". El primero se dedica al uso de la contabilidad en materia judicial, mientras que el segundo se dedica al estudio de la información financiera para detectar fraudes. Ambas actividades se han convertido en un negocio de consultoría para muchas firmas de servicios profesionales, incluidas las firmas de auditoría.

\footnotetext{
${ }^{3}$ The Center for Audit Quality (CAQ), es una organización política pública autónoma dedicada a
} mejorar la confianza de los inversores y la confianza pública en los mercados mundiales de capital. 
En las últimas dos décadas hemos asistido a un gran número de casos de fraude, pero quizás si uno ha resultado paradigmático y ha tenido unas repercusiones significativas, ese ha sido el caso Enron. Tras Enron se produjo una fuerte reacción legislativa en EE.UU., la Ley Sarbanes-Oxley (SOX) abrió el debate sobre el modelo contable estadounidense tachado de estar basado en reglas y no en principios y la desaparición de Andersen, una de las entonces "Big Five" firmas de auditoría. Este último efecto fue fruto de un segundo fraude vinculado al anterior, el fraude de Andersen. El auditor responsable destruyó las pruebas de su conducta y el fallo del control en la Firma llevó a que se le retirase su licencia para auditar entidades supervisadas por la Securities and Exchange Commission (SEC). Tres años después, recurrida aquella decisión, se dio la razón a Andersen, pero ya era tarde, los socios de la firma y sus equipos habían iniciado un éxodo hacia otras firmas y ya no quedaba nada (Sauviat, 2006).

La prevención del fraude exige como primera aproximación entender por qué se produce; a continuación evaluar en qué medida los mecanismos institucionales deben ser perfeccionados y si no se requieren nuevos mecanismos $y$, finalmente, evaluar por qué fallan los existentes y tomar medidas destinadas a mejorar su eficacia.

La explicación de la conducta fraudulenta se ha realizado en torno al conocido modelo del triángulo, planteado por el criminólogo Donald Cressey, que encuentra tres condiciones que deben estar presentes en un fraude: un incentivo o presión, la oportunidad para cometer fraude y una racionalización de la conducta (Kassem y Higson, 2012).

El incentivo o presión puede proceder del propio modo de vida del defraudador o de las presiones de la propia organización. Pueden ser de naturaleza no financiera (por ejemplo, adicciones, falta de disciplina personal, satisfacción del ego, reputación o presión social) o financiera (problemas económicos repentinos, deudas de juego, estructura salarial o bajo salario, problemas financieros para la entidad). La oportunidad viene determinada por los factores del entorno que permiten el fraude o que, en caso de producirse, impiden que sea descubierto. Ningún defraudador comete el ilícito si sabe que va a ser descubierto. La oportunidad es especialmente relevante en las organizaciones con un control interno deficiente. Asimismo, el modelo del triángulo también explica que el fraude de gran envergadura se cometa en los niveles más altos de la organización, dada su capacidad para sortear los controles o para disuadir a los subordinados en el ejercicio eficaz de sus funciones de control. Finalmente, la racionalización que realiza el defraudador le permite crear una estructura ética, una estructura axiológica propia, donde su conducta no es reprochable. Así, se falsearán cuentas para evitar un mal mayor como el cierre 
de la empresa, o para evitar el despido, porque no se me retribuye equitativamente o de acuerdo al valor que aportan mis decisiones o porque como todo el mundo lo hace, yo no voy a ser el tonto.

El modelo del triángulo de Cressey es la columna vertebral de las mejores prácticas en la auditoría destinada a descubrir fraudes, la International Auditing Standard 240. Este modelo ha inspirado algunas variantes, como el modelo del diamante, en el cual, además de las tres condiciones enunciadas, se une la competencia del defraudador (Wolfe y Hermmanson, 2004). La competencia del defraudador es la oportunidad que tiene que ver con el conocimiento o posición del defraudador. Esta variante ayuda a distinguir las oportunidades que están presentes en la organización de las que se dan cita en la persona del defraudador.

Otras variantes al modelo se sitúan en el terreno de la racionalización y tienen que ver con el nivel ético preexistente, también denominada integridad, que impida la formación de escalas axiológicas "ad hoc", destinadas a dar cobijo moral a la actitud ilícita. La integridad sería más evaluable a efectos de identificación de factores de riesgo de un fraude (Albretch, 1984)

Las tres motivaciones de Cressey deben estar presentes para la comisión del fraude. En consecuencia, existen tres frentes para mitigar el nivel de riesgo de que se incurra en un fraude. La conceptualizacion de los condicionantes de la conducta fraudulenta permite una revisión de las medidas a tomar para mitigar un problema que mina uno de los pilares del sistema financiero: la confianza.

En este documento presentamos las principales actuaciones relativas a dificultar la comisión de fraudes. En los últimos años ha habido una proliferación regulatoria, como consecuencia de la necesidad social de mejorar el sistema financiero, lo que es buena muestra de la relevancia de la cuestión. Los escándalos financieros son uno de los frentes donde se requiere evaluar si el déficit es estructural o el sistema puede ser mejorado para extirpar unas prácticas tan nocivas.

El capítulo segundo lo destinaremos a revisar posibles actuaciones para mitigar los incentivos que mitiguen el riesgo de fraude. En el epígrafe tercero tratamos las actuaciones para evitar las oportunidades y en el cuarto las dirigidas a la generación de un clima de integridad que dificulte la racionalización de las conductas fraudulentas. Finalizamos con las conclusiones en el punto quinto. 


\section{Actuaciones para mitigar los incentivos en la comisión de fraudes}

La lógica del mercado empuja ineludiblemente a la evaluación del desempeño en función de los resultados. El capital no entiende de otros criterios, porque lo que aspira es a la mayor rentabilidad posible para un nivel de riesgo determinado. En una entidad capitalista el resultado es el "rey", el cómo, siendo lícito, pasa a un lugar secundario. En un estudio de la firma de auditoría, KPMG, a unos 5.000 mandos intermedios en EE.UU., observaron que el $59 \%$ habían recibido presiones para alcanzar los resultados y que el $52 \%$ pensaban que son valorados por sus buenos resultados y no por los esfuerzos que realizan (CAQ, 2010).

Esta presión sobre los primeros directivos de la organización se traspasa, como correa de transmisión, a toda ella, generando una cultura resultadista por conseguir los objetivos marcados. La sanción puede ser desde una menor retribución al despido. En Enron, por ejemplo,

la cultura era de competencia destructiva. Aquéllos cuyo desempeño se juzgaba en el quintil más bajo eran despedidos, incluso si evaluados objetivamente hubieran sido considerados como competentes. Esto orientó la atención de los jóvenes directivos no tanto a conseguir objetivos valiosos, sino a satisfacer a sus superiores y denostar a sus colegas (Kennedy, 2006: 216).

El lema de Enron era: Respect, Integrity, Communication and Excellence (RICE); sin embargo bien pudiera haber sido: Risk-taking, Individualism, Contemptand Exploitation (Arriesgada, Individualista, Arrogante y Explotadora) (Dobson, 2006: 195).

En este artículo vamos a analizar tres prácticas que son consecuencia de la necesidad de financiar empresas de gran envergadura. La primera de ellas es la necesidad de los propietarios de confiar la gestión a un equipo de directivos conforme una empresa gana dimensión; entre otras razones por la complejidad asociada a la dimensión y porque muy previsiblemente la base de propietarios se amplía. Como consecuencia de esta separación es preciso articular esquemas retributivos que alineen los intereses de los gestores y los propietarios. La segunda se refiere a la necesidad de importantes volúmenes de financiación con plazos largos o en proyectos que se van a abordar y sobre los que la incertidumbre del prestamista es mayor. En estos casos, se establecen cláusulas que permiten la monitorización continuada de dichas financiaciones de plazo largo o con niveles más elevados de riesgo. La tercera práctica es la importancia que adquieren en el capitalismo moderno, ágil y global, las firmas dedicadas a asesorar a los inversores en sus decisiones dotando a estas firmas de una gran influencia en las empresas debido al efecto de sus recomendaciones a los inversores. 
Los esquemas retributivos tratan de alinearse con los intereses de los propietarios para reducir los costes de agencia; ineludiblemente aumentan los riesgos de fraude. En ese entorno, ¿̇los accionistas saben que están incrementando el riego de fraude en la información? Una cultura agresiva hacia los resultados alimenta un mayor riesgo de comisión de fraude. ¿̇Se puede salir de la competencia por mayores rentabilidades? La respuesta es compleja y depende de los niveles de dependencia de financiación que tenga la entidad. Conforme aumenta su dependencia, o el núcleo accionarial esté más disperso, provoca que la entidad esté sometida a la dinámica competitiva y a introducir mayor presión sobre las cifras. En otro caso, la inversión en esas acciones no parece, en términos comparativos con otras compañías, tan atractiva.

Por otra parte, la financiación procedente de las entidades de crédito suele estar sometida al cumplimiento de determinados ratios durante la vigencia de los contratos, cuyo incumplimiento podría activar una devolución anticipada (esta condición estaría comprendida entre los conocidos como "covenants"). Ante periodos de caída de la actividad estos "covenants" son auténticas "espadas de Damocles" que penden sobre la financiación de las empresas, suponiendo una seria amenaza de entrar en suspensión de pagos y concurso de acreedores. Este escenario favorece la manipulación de las cifras y la presión sobre los responsables de la elaboración de la información y su auditoría con la finalidad de presentar una situación que permita eludir un eventual incumplimiento del "covenant". Sin embargo, estas cláusulas tienen una legitimidad clara, son las medidas que toma el financiador para conseguir la recuperación de su inversión ante un proyecto empresarial que se ha tornado con mayor riesgo que el inicialmente evaluado. El problema reside cuando el nivel de endeudamiento es excesivo. Realmente, ¿̇controlan los propietarios estos niveles de riesgo que proponen sus directivos cuando no están en el día a día de las operaciones?

El papel de las casas de análisis de valores es creciente pues efectúan recomendaciones a sus clientes sobre las oportunidades de inversión o aconsejan la desinversión. Las entidades también son seguidas y evaluadas por las agencias de calificación crediticia que informan sobre su calidad crediticia. Asimismo, han surgido nuevas entidades destinadas a asesorar a los inversores en el uso de sus derechos políticos, son las "proxy advisors" que emiten recomendaciones sobre el sentido del voto en las juntas generales de las entidades que siguen. El panorama de "stakeholders" interesados en conocer la información de la entidad se ha ampliado. Por otra parte, la globalización de los mercados, así como la complejidad que implica el análisis de la información elaborada por las entidades emisoras de valores, dota de un gran valor para los usuarios a estos intermediarios. Las entidades emisoras 
de valores negociados articulan departamentos, como el de relaciones con los inversores, que tratan de mantener informada en condiciones de igualdad a este grupo de "stakeholders", así como de informar regularmente a las autoridades del mercado. El efecto de las "malas" noticias es fulminante en la cotización de los valores de la entidad y esta exposición tan sensible es un factor que introduce un riesgo de maquillaje en la información que se comunica.

Tanto los esquemas retributivos ligados al desempeño, como los "covenants" son dos prácticas útiles para reducir los problemas de agencia, el primero, y la asimetría de información en el segundo, pero que deben ser monitorizados. A ellos uniremos un tercero que es el interés por ganarse el favor de las casas de análisis que son las que orientan la inversión en los mercados.

El riesgo asociado a los esquemas retributivos ligados al desempeño es el trasvase de rentas de los propietarios a los directivos. Erickson et al (2004) han analizado 50 compañías que cometieron algún fraude entre 1966 y 2000 observando que éstas presentaban una mayor probabilidad de presentar esquemas de retribución vinculados a la cotización (una de las formas de medir el desempeño). Los propietarios pueden mitigar este riesgo desde dentro de la organización y desde fuera. Desde dentro, a través del refuerzo del consejo de administración y sus comisiones delegadas, en concreto las comisiones o comités de auditoría y de retribuciones; desde fuera, mediante la auditoría externa de sus estados financieros.

La comisión de auditoría es una comisión delegada del consejo de administración, obligatoria en España para las sociedades con títulos admitidos a cotización oficial, compuesta por consejeros no ejecutivos (los que no tienen funciones de gestión), normalmente por externos (de reconocido prestigio) o por dominicales (en representación de los propietarios). Para la comisión de auditoría se busca que los consejeros externos tengan un fuerte conocimiento de los fundamentos económicos del negocio y de los riesgos que se pueden derivar, así como al menos uno de ellos disponga de profundos conocimientos en materia de contabilidad y auditoría (Directiva 2014/56). Su misión es velar por la calidad de la información que se eleva a los accionistas, el buen funcionamiento del control interno de la entidad y mantiene, en el ejercicio de esta misión, una relación privilegiada con los auditores externos, y lo ideal sería que dependiese de esta comisión el departamento de auditoría interna, aunque no siempre es así. Un eficaz funcionamiento de la comisión de auditoría, con consejeros independientes y capaces, y con recursos como el departamento de auditoría interna, permite reducir el riesgo de manipulación en las cifras para conseguir el cumplimiento de los objetivos. La cuestión de los esquemas retributivos a la dirección es un factor tan relevante que el propio $C A Q$ 
(2010) indica que comienza a ser frecuente la coordinación entre la comisión de retribuciones y la de auditoría en esta materia, tan ligada al ámbito competencias de la primera.

El segundo de los riesgos apuntados, la existencia de "covenants", suele ser vigilado por el auditor externo. Al auditor externo lo nombra la entidad auditada, lo cual le resta de partida un ápice de independencia, sin embargo, ésta trata de restaurarse con un exigente mecanismo de responsabilidad ante terceros por su actuación negligente. Las firmas de auditoría son entidades de aseguramiento, tanto más confiables cuanto más profundos sean sus bolsillos ("deep pocket" fue un término acuñado para definir su papel), siendo frecuente que ante contratos relevantes, las entidades financieras exijan una compañía de las denominadas "Big Four", por su especialización en la auditoría de grandes compañías y por su capacidad para asumir la reparación de un quebranto. El Reglamento 537/2014 considera nulas de pleno derecho dichas cláusulas en aras de no limitar el derecho de elección que le corresponde a la Junta General de accionistas y en aras también de dinamizar el mercado de auditoría de las entidades de interés público, dominado por las "Big Four".

El tercero de los riesgos planteados es la presión que sufren las empresas por conseguir sus objetivos de beneficios, dividendos, crecimiento o cumplimiento de previsiones. En este caso, el fraude se comete sobre los futuros inversores, al hacerles pensar que la entidad está obteniendo mejores desempeños que los reales. Al igual que en el caso de los "covenants", es la auditoría externa la primera trinchera donde luchar contra el riesgo de fraude, pero en este caso, dada la quiebra de la confianza en el mercado en general, son las entidades supervisoras del mercado, la Securities and Exchange Commission (SEC) en Estados Unidos, o la Comisión Nacional del Mercado de Valores (CNMV) en España, las que asumen la responsabilidad de evitar que en su mercado circule información fraudulenta. El reciente caso de Gowex es un ejemplo de fraude en la información que ha puesto en jaque la credibilidad de un mercado como el Mercado Alternativo Bursátil (MAB) y que ha provocado como primera reacción que todas las entidades del mercado hayan contratado a firmas del grupo "Big Four" y que se esté demandando la revisión de los requisitos de información exigidos a estas compañías, además de un fuerte cuestionamiento de la labor de protección del inversor que tienen los supervisores del mercado.

Beasley et al (2010), en el estudio auspiciado por el COSO, señalan que los motivos con más influencia en el fraude fueron: la presión por conseguir las expectativas de ganancias, la necesidad de esconder la deteriorada situación financiera, mejorar el precio de las acciones, facilitar la financiación y mejorar los planes de retribución. 


\section{Actuaciones para evitar las oportunidades en la comisión de fraudes}

La oportunidad tiene lugar porque la persona o personas con capacidad para defraudar se encuentran en la posición adecuada y en una organización que no establece los mecanismos suficientes para evitar el fraude. En el estudio de Beasley et al (2010) se pone de manifiesto que este riesgo se da cita especialmente en las personas del primer ejecutivo y del director financiero; en un $89 \%$ de los casos uno de los dos o ambos intervinieron en el fraude.

El riesgo derivado de las oportunidades puede ser mitigado mediante mecanismos preventivos o a través de actuaciones de detección de los fraudes. Estas actuaciones deberían propiciar entornos lo más seguros posibles y extremar la precaución y el escepticismo en las personas que pueden cometer el fraude.

Las oportunidades de cometer fraude en la información se incrementan cuando la entidad opera en un tipo de actividad en el que la elaboración de la información financiera requiere realizar numerosas estimaciones. Esto suele suceder en compañías con largos ciclos de realización en los que es preciso estimar si los recursos se convertirán finalmente en dinero para la compañía, por ejemplo, en la actividad de intermediación financiera la duda reside en estimar si los créditos concedidos resultarán morosos. La elaboración de información financiera no es una técnica mecánica y automática, es preciso aplicar fuertes dosis de criterio profesional para conseguir ofrecer una imagen fiel de la situación financiera del patrimonio y de los resultados obtenidos. Adicionalmente, las normas contables hasta hace una década eran un mosaico a nivel internacional, con diferencias que ponían de manifiesto lo relativo del resultado obtenido con la aplicación de determinada legislación contable. Actualmente, existe un sólido proceso de armonización internacional, en torno a una institución internacional (el International Accounting Standards Board -IASB-I, en el que además se han reducido, prácticamente hasta su desaparición, los tratamientos contables alternativos. Estos tratamientos contables alternativos posibilitaban que dos empresas, ante una misma transacción, ofreciesen cifras diferentes, sin dejar de cumplir ninguna de ellas el cuerpo regulatorio.

A propósito del escándalo de Enron ha saltado al debate, especialmente en EE.UU., que las normas contables de aquel país estaban basadas en reglas y no en principios lo que implicaba que el sistema contable no era teleológico, por lo que la aplicación de las normas, muy detalladas, debería conducir a una imagen fiel, sin realizar un análisis del fondo económico de las transacciones. Sin embargo, este grado de detalle, que pretendía mitigar los efectos del criterio profesional al 
elaborar la información, se volvía en contra pues los contratos se diseñaban para eludir las prescripciones contables, si bien el espíritu de los contratos y su racionalidad económica era diferente. Por ejemplo, muchas compañías estadounidenses conseguían sacar de su balance los compromisos no cancelables contraídos por contratos de arrendamiento, simplemente estableciendo plazos justo por debajo del $75 \%$ de la vida útil del recurso arrendado. De esa manera, la obligación no se reconocía, con la consiguiente mejora del ratio de endeudamiento que pone en relación la proporción de deuda sobre los fondos propios de la entidad.

El entorno de confianza se consigue implantando sistemas que separen nítidamente las funciones de gobierno y de gestión, confiriendo a los órganos encargados del gobierno la misión de controlar los riesgos de la entidad entre los que se encuentran el buen funcionamiento del sistema de control interno. En 2002, la ley SarbanesOxley, principal reacción legislativa en EE.UU. a los escándalos financieros de comienzos del siglo XXI, extremó los requerimientos a los administradores sobre el funcionamiento del control interno, obligándoles a evaluar el funcionamiento del control interno y a auditar externamente el ejercicio de dicha responsabilidad. En España, la reforma de la Ley del Mercado de Valores del año 2002 (a través de la Ley 44/2002, de medidas de Reforma del Sistema Financiero) obliga a la constitución de los comités de auditoría y con especial énfasis desde la reforma introducida por la Ley 12/2010 por la que se modifica la Ley19/1988, de Auditoría de cuentas, que introduce la obligación de dichos comités de supervisar el funcionamiento de los sistemas de control interno y de los sistemas de gestión de riesgos, así como los procesos de emisión de información financiera. El proceso normativo anterior ha venido seguido de la modificación del contenido del informe de gobierno corporativo, incluyendo un apartado en el que los administradores explican el sistema de control interno y las medidas de gestión de riesgos en relación con la emisión de información financiera (Ley 2/201 1, de Economía Sostenible), siendo revisada dicha declaración por los auditores externos. En 2013, la Circular 5/2013 de la CNMV ha modificado el modelo de informe de gobierno corporativo para incluir los avances anteriores. Recientemente, se ha aprobado la Ley de Sociedades de Capital donde se legisla sobre las comisiones delegadas del consejo de administración, estableciendo la obligatoriedad de la comisión de retribuciones y nombramientos y requiriendo que esta comisión y la comisión de auditoría esté formada por consejeros no ejecutivos y estén presididas por un consejero independiente.

La tradicional rendición de cuentas que ejercen los administradores sobre los directivos suponía un control del desempeño de las entidades. La información financiera permitía evaluar la gestión desarrollada y los riesgos incurridos para 
alcanzar los resultados. Se trata de un control "a posteriori" que puede resultar tardío, si los riesgos asumidos se hubiesen materializado de manera negativa para la entidad. Uno de los problemas de la relación de agencia es el riesgo moral. Los recursos asignados a los gestores (agentes) pueden ser aplicados a proyectos más arriesgados que el perfil deseado por los propietarios (principales), dado que el incremento de riesgo, aumenta las posibilidades de ganancia, manteniendo el nivel de pérdidas para el gestor en el mismo nivel. La exigencia de responsabilidad sobre la gestión de riesgos permite controlar el riesgo moral en el que pueden incurrir los gestores de la entidad. La evaluación del desempeño que se desprende de la lectura de los estados financieros se efectuaría en un entorno de riesgo asumido, mientras que la política de riesgos de los directivos no se analiza hasta que se conocen sus consecuencias y no antes de abordarlos.

Esta importante función asignada al comité de auditoría podrá ser desempeñada eficazmente si cuenta con los recursos necesarios para desarrollarla. Básicamente son dos los instrumentos con los que cuenta: los departamentos de auditoría interna y la selección y seguimiento del trabajo de los auditores externos.

El primero es el departamento de auditoría interna, órgano encargado de revisar el funcionamiento del control interno. El departamento de auditoría interna realiza el trabajo de campo sobre el que el comité de auditoría es responsable último, por lo que la dependencia del comité de auditoría sería la mejor forma de garantizar su independencia. Durante muchos años, se ha tratado de dotar de valor añadido a la función de auditoría interna, siendo empleado como departamento para el desarrollo de proyectos especiales, encargados por la alta dirección. Sin embargo, este rol introduce un sesgo en el departamento encargado de velar por la buena marcha del sistema de control interno, pues pierde independencia si debe evaluar su propio trabajo. La contribución valiosa del departamento de auditoría interna, en un marco de separación de gobierno y gestión, reside en ser el instrumento operativo de una de las responsabilidades de gobierno.

El segundo instrumento es la selección y seguimiento del trabajo de los auditores externos. El nombramiento de los auditores externos por parte de un comité donde no participan los directivos, es una forma de garantizar la independencia de los auditores frente a la Alta Dirección. La comunicación continuada permite identificar las políticas contables discutibles, los indicios de fraude y coordinar los instrumentos para soportar las acciones a adoptar.

La función de auditoría externa es valiosa porque se sustenta en dos atributos: la competencia y la independencia. Si faltan ambas, la función es inútil. Una relación 
prolongada entre el auditor y la firma auditada cuestiona la independencia de este último por lo que se ha debatido sobre la conveniencia de exigir la rotación de auditores tras un determinado periodo de años. En España, la historia de nuestra regulación sobre el particular ha ido desde unas previsiones muy restrictivas al promulgarse la Ley de Auditoría de cuentas en el año 1988, que situaba la duración del contrato entre un mínimo de tres años y un máximo de nueve, imponiendo la rotación de la firmas de auditoría. En 1995, con motivo de la aprobación de la Ley de Sociedades de Responsabilidad Limitada, se incluyó una disposición adicional que permitía la renovación por periodos máximos de tres años, una vez alcanzado el máximo de nueve años. La proximidad del primer ciclo de nueve años, y la conmoción que se produciría en el mercado de la auditoría, especialmente entre las entonces "Big Six", pudieron tener alguna influencia en esta modificación legislativa. El siguiente episodio tuvo lugar en 2002, con motivo de la Ley Financiera, en plena vorágine de escándalos financieros. En ella se incorporó la obligación de rotación del auditor firmante y su equipo, en la auditoría de sociedades cotizadas o de gran dimensión, si bien la firma podía mantener el contrato. Esta previsión se ha mantenido en 2010 con motivo de la reforma de la ley de auditoría de cuentas. En 2014, el Parlamento Europeo ha aprobado el Reglamento 537/2014 aplicable a las entidades de interés especial en la que es más exigente en cuanto a la rotación de los auditores. El periodo máximo será de diez años, pudiendo renovarse por un periodo de otros diez años si la renovación se ha sometido a un concurso público de ofertas o de catorce años si es en régimen de coauditoría (régimen vigente en Francia).

El cambio de auditores externos es más elevado en las compañías que cometen fraudes. Beasley etal (2010) observaron una diferencia significativa entre compañías que cometieron fraudes y una muestra de control que no lo habían cometido. La compra de opinión es un fenómeno estudiado en la literatura sobre auditoría. Por ejemplo, Pescanova el año anterior al descubrimiento del fraude había cambiado de auditor.

Otro riesgo que también cuestiona la independencia de los auditores es la relación comercial con el cliente de auditoría. En España, entre otros países, se considera una amenaza a la independencia la circunstancia de que un cliente de auditoría absorba más del 15\% de los honorarios debiendo buscar el auditor medidas que palien esta dependencia. En el caso Enron se puso de manifiesto que el excesivo peso de los socios regionales, los de la línea de negocio, sobre la estructura corporativa impidió el funcionamiento de los mecanismos de control de la firma de auditoría, uniendo al fraude de la compañía, el fraude en la firma de auditoría. Un año antes, uno de los socios responsables de la supervisión fue objeto de 
fuertes críticas por parte de los directivos de Enron y del equipo de auditoría, que terminaron con el cese en estas responsabilidades (Sauviat, 2006). Según describe Sauviat (2006), los socios cobraban en función de sus facturaciones, lo cual puede suponer una fuerte presión sobre un socio al afectar seriamente a sus rentas y estimular un comportamiento individualista frente al estímulo de mantenimiento de la reputación que es institucional.

Este riesgo se ha intensificado por las voluminosas facturaciones que se realizan a los clientes de auditoría en servicios de consultoría. Estos servicios son contratados por la Alta Dirección y esta situación de por sí introduce un conflicto de interés en quien posteriormente debe emitir un informe sobre los estados financieros que evalúan a esa Alta Dirección ante sus órganos de gobierno. La Directiva Europea 2014/56 requiere que el comité de auditoría autorice la contratación de los servicios de no auditoría con el auditor legal.

Sin embargo, el debate no es tan sencillo tal como incompatibilizar la prestación de otros servicios. En las últimas tres décadas se han producido importantes procesos de concentración en el mercado de la auditoría. Como dice el profesor Zeff (2003) las firmas de auditoría hoy son grandes empresas, que desarrollan negocios lucrativos en consultoría además de la auditoría. Este crecimiento de las organizaciones de control les confiere una mayor percepción del riesgo reputacional, en caso de mala praxis, especialmente desde la desaparición de Andersen en 2002 con motivo del escándalo Enron. Por otra parte, este crecimiento les permite mejores retribuciones a sus profesionales y contar con equipos que puedan mantener un nivel de competencia adecuado a la hora de enjuiciar los poderosos equipos técnicos de las grandes compañías. Adicionalmente, aunque las prescripciones éticas en determinados casos impidan la incorporación a las compañías auditadas en un periodo de tiempo, existe el riesgo de que la renuncia a negocios lucrativos suponga una dificultad añadida a la retención del talento.

La regulación ética desde la IFAC ${ }^{4}$, así como las prescripciones legislativas a ambos lados del Atlántico desde inicios del siglo XXI han incrementado las incompatibilidades en este terreno. La reciente directiva sobre auditoría (Directiva 2014/56 de 16 de abril de 2014, por la que se modifica la Directiva 2006/43 relativa a la auditoría legal de las cuentas anuales y de las cuentas consolidadas), sobre la que ya está trabajando el Gobierno de España para su incorporación en la

${ }^{4}$ The International Federation of Accountants (IFAC) es la organización mundial de la profesión contable dedicada a servir al interés público fortaleciendo la profesión y contribuyendo al desarrollo de la economía internacional. Está compuesta por 179 miembros y asociados en 130 países y jurisdicciones. 
legislación nacional, prevé una identificación más nítida de los servicios que se pueden prestar por parte de los auditores.

Los datos revelados en EE.UU. han puesto de manifiesto una mayor incidencia del fraude en la información en entidades auditadas por firmas pequeñas que en las auditadas por la "Big Four" o las 4 grandes firmas nacionales (Beasley et al, 2010).

Con todo, solo en el $23 \%$ de los casos la SEC encausó a los auditores de las compañías que cometieron fraude y fueron sometidas a un procedimiento sancionador por tal motivo (Beasley et al, 2010). La finalidad de la auditoría no es la detección de cualquier fraude sino de establecer un conjunto de procedimientos que confieran una seguridad razonable de que los estados financieros se hayan libres de errores significativos que pudiesen alterar la opinión de un tercero sobre la entidad. En el caso del fraude la actuación del defraudador convierte en más improbable su descubrimiento que en el caso de un error no intencionado, por lo que el nivel de seguridad es menor. No es misión de la auditoría de estados financieros el descubrimiento de cualquier tipo de fraude, si bien el auditor debe extremar su escepticismo profesional ante indicios de fraude.

En caso de detectarse un fraude surge un debate ético relevante. La auditoría de cuentas se fundamenta en el secreto profesional. El secreto profesional es una institución presente en ciertas profesiones, entre ellas la auditoría. La definición del alcance del secreto profesional en el ámbito de la auditoría también ha ido evolucionando hacia posiciones de mayor responsabilidad social de esta profesión. El descubrimiento de un fraude afectará a la opinión del auditor y supondrá la obligación de informar internamente a los niveles organizativos que puedan tomar las acciones oportunas y en determinadas jurisdicciones, obviar el deber de secreto profesional y denunciarlo a las autoridades supervisoras competentes o a las judiciales. En España, existe el deber de información a las autoridades supervisoras cuando la entidad auditada maneje fondos de terceros con carácter fiduciario y siempre que comprometan la legalidad de las mismas, su continuidad - afecte seriamente a la opinión de auditoría (artículo 25 de la Ley de Auditoría de Cuentas). A las instancias judiciales lo prevé el artículo 262 de la Ley de Enjuiciamiento Criminal.

Un último mecanismo consiste en el establecimiento de "programas de informadores" de fraudes ocurridos dentro de la organización. La figura del informador ("whistleblowing" en inglés) ha sido ampliamente tratada en los ambientes anglosajones. La ley Sarbanes-Oxley ya apunta entre sus recomendaciones el establecimiento de programas para esta figura que les garanticen que no van a sufrir represalias. 
$C A Q(2010)$ recomiendan que estos programas dependan del comité de auditoría y que para incentivar su funcionamiento deben investigarse las informaciones de manera rápida, imponiendo las sanciones cuando las evidencias son confirmadas. La relación entre información, investigación y sanción genera un ambiente positivo para que se fortalezca el tono ético de la organización (Mesmer-Magnus y Viswesvaran, 2005). No obstante, este documento alerta sobre la principal debilidad que no es otra que las garantías conferidas al informante sobre la confidencialidad, y evitar las represalias, así como la desconfianza en que la Alta Dirección tome medidas ante los comportamientos poco éticos. La literatura científica no ha podido demostrar qué factores inducen a revelar un comportamiento fraudulento, especialmente los situados al nivel del individuo. Esta información sería de gran interés para el diseño de programas de concienciación en ese grupo de individuos. Sin embargo, al nivel de la organización, se ha conseguido evidencia consistente de que en las entidades en las que existe un fuerte apoyo a esta práctica por parte de la Alta Dirección, como en las que existe una cultura o ambiente de trabajo en equipo, amigable y con un fuerte componente ético, reportaban en mayor medida el fraude (Mesmer-Magnus y Viswesvaran, 2005; Vadera et al, 2009).

El difícil papel de los informadores puede verse en los largos años que dedicó Harry Markopolos a denunciar el esquema piramidal de Bernard Madoff, desde 2000 a 2009, o por ejemplo la reacción inicial al informe de Gotham Research en el reciente escándalo de la firma Gowex en la que se acusó a esta casa de análisis de estar movida por intereses especulativos. $Y$ estos dos casos son informadores externos a la entidad y en los que las represalias son menores.

Los mecanismos anteriores serían útiles para disminuir el riesgo de comisiones de fraude de los directivos con sus accionistas, sin embargo no evitan el riesgo de fraude de la información de la entidad con futuros inversores. En estos casos, los guardianes pasan a ser los auditores externos, como actividad de interés público y con responsabilidad ante los terceros que pudieran verse perjudicados por el informe de auditoría $y$, en el caso de los mercados de valores y financiero, las entidades supervisoras (en España la CNMV y el Banco de España, respectivamente). El supervisor bancario sí asume funciones de inspección de la información, pues un fraude en la misma puede ocultar los riesgos que genera al sistema una entidad concreta; por su parte el supervisor del mercado de valores cimenta la confianza en la información en el mecanismo de la auditoría externa. Precisamente, esta colaboración tan estrecha con los auditores externos les lleva a encargarles informes especiales de consultoría, como el análisis de la salida a Bolsa de un nuevo emisor. Este encargo en el caso de la salida a Bolsa de Bankia y el encargo a Deloitte, también su auditor legal y quien a juicio del supervisor era el más idóneo 
para hacerlo por el conocimiento sobre la entidad y el sector, ha sido cuestionada por el Instituto de Contabilidad y Auditoría de Cuentas como una quiebra en la independencia del auditor legal (Diario Expansión, 2014).

La responsabilidad de los órganos supervisores también consiste en crear un entorno que potencie el establecimiento de sólidos mecanismos de gobierno corporativo, entre ellos, exigir la divulgación de las prácticas de gobierno corporativo de manera normalizada, así como iniciar procedimientos sancionadores a las entidades emisoras como a sus auditores en caso de la detección de fraudes. La actuación del supervisor es posiblemente más eficaz señalando públicamente a los infractores en un registro de infracciones que imponiendo sanciones pecuniarias (Cañibano y Pedrosa, 2008).

Finalmente, en la comisión de fraudes que impliquen apropiación de bienes, el defraudador deberá lavar el producto de su ilícito y para ello, las mejores prácticas internacionales recomiendan a los Estados que impongan a su sector financiero la obligación de comunicar las transacciones sospechosas de ser lavado de activos o perseguir la financiación del terrorismo. Las recomendaciones del Grupo de Acción Financiera Internacional (GAFI), suscritas en lo que afecta al sector financiero por el Comité de Basilea, han generado todo un cuerpo internacional que coordina las actuaciones a nivel global. Ante transacciones sospechosas, las entidades financieras y otros sujetos obligados deben informar a la autoridad competente, en España el Servicio Ejecutivo de Prevención del Blanqueo de Capitales (SEPBLAC). En 2010 se aprobó la Ley de prevención del blanqueo de capitales y de financiación del terrorismo, siendo en mayo de 2014 cuando se ha aprobado el Reglamento que la desarrolla.

Por otra parte, quizás uno de los mayores retos lo constituye la existencia de paraísos fiscales, en los que no se declara el origen de los fondos depositados y representan la vía más frecuente para poner a buen recaudo los mismos. La actuación de los informadores ha permitido destapar fondos depositados por evasores fiscales y ha provocado demandas contra las entidades financieras depositarias de los fondos por revelación del secreto bancario.

Como reflexión final sobre los incentivos, recogemos las ideas de Boatright (2006), a propósito de Enron. El autor realiza un certero análisis sobre la naturaleza de los mecanismos con los que se regula la relación de agencia. El sistema de controles está fundamentado en instituciones (agentes) que contraen obligaciones fiduciarias con terceros y actúan velando por el interés de terceros (principal), como por ejemplo los directivos de una compañía actúan en interés de los propietarios, los 
auditores con el público en general o la banca de inversión con los pequeños inversores a los que confieren servicios de asesoramiento para poder integrar la información existente en el mercado sobre las oportunidades de inversión. Este modelo de control de las relaciones se fundamenta en un fuerte deber moral del agente con su principal. Sin embargo, se han introducido paulatinamente mecanismos de mercado que tratan de buscar los óptimos en las relaciones como la retribución basada en el desempeño de los directivos; el aumento de los servicios profesionales que prestan las firmas de auditoría a sus clientes, incorporando negocios más lucrativos como la consultoría, que les permitía también atraer mejores profesionales; o la consolidación de la actividad de asesoramiento a inversores con los servicios a grandes corporaciones que ha experimentado la banca de inversión. Estos mecanismos fallan cuando a los directivos se preocupan por incrementar el precio de las acciones en el corto plazo, a los auditores les pesa más satisfacer a los directivos de las empresas a auditar (porque después les contrataban los servicios de consultoría) que al público al cual sirven, y a la banca de inversión está más preocupada en las operaciones empresariales que en el servicio de asesoramiento a la clientela a la cual inundaban con recomendaciones viciadas por sus propios intereses. El conflicto de interés ha emergido como un inhibidor de los mecanismos fiduciarios establecidos en el sistema financiero. La regeneración ética comienza por limitar los conflictos de interés y por exigir una mayor salvaguarda de las obligaciones fiduciarias

\section{Actuaciones contra la racionalización de la conducta fraudulenta}

El defraudador necesita justificar su actuación en la necesidad, personal o de la entidad, o en el carácter esporádico de su actuación. La racionalización del comportamiento del defraudador es un mecanismo justificativo que se desenvuelve en personas con una débil capacidad de discernimiento o con un nivel de integridad precario. El clima de integridad es un elemento de la cultura que ha de ser promovido desde la Alta Dirección, no sólo con mensajes, sino también con acciones (CAQ, 2010). Si los máximos responsables se someten a estrictas normas éticas, la racionalización del fraude disminuye.

La integridad supone ponderar en mayor medida los medios que los fines; en un entorno íntegro no todo vale para conseguir el resultado. Las organizaciones muy orientadas al resultado presentan un riesgo más elevado de justificar las conductas fraudulentas o delictivas en general. En un estudio reciente de la firma de auditoría 
y consultoría EY $(2014)^{5}$, han observado que el $42 \%$ de los directivos entrevistados, alrededor del mundo, y dentro de éste el $46 \%$ del subgrupo de directores financieros justificarían la comisión de actividades ilícitas o inmorales para hacer negocios, como ofrecer entretenimiento, hacer regalos, pagar comisiones o falsear los estados financieros. Los datos son preocupantes. En concreto, el falseamiento de cifras sería aceptable para un $6 \%$ de los ejecutivos entrevistados.

En ocasiones, los protagonistas de los escándalos más sonados en EE.UU. estaban embarcados en múltiples actividades filantrópicas lo que ha servido para construir un discurso aparente de integridad (Jennings y Hapel, 2006), al tiempo que sometían la cultura corporativa a una fuerte tensión cortoplacista. Las actividades filantrópicas son loables pero forman parte de una integridad exhibida en lugar de la integridad exigible en sus modos de proceder y que pasan ineludiblemente por una política de transparencia efectiva.

Una segunda cuestión es la fuerza que ejerce la obediencia debida como mecanismo que inhibe la denuncia de las conductas fraudulentas en las más altas instancias. Como señalan Gonzalo y Garvey (2007) en muchas ocasiones el administrador que tiene responsabilidad sobre las cuentas, pero que aduce desconocer la técnica, presiona al responsable de preparar los estados financieros, que tienen conocimiento pero no responsabilidad, para que las "cocine" a su gusto. Argumentos como "los números tienen que salir como sea" o "lo que no está prohibido está permitido" son líneas discursivas que conducen inevitablemente al fraude en la presentación de una imagen fidedigna de la entidad. En caso de litigio, el administrador alegará que él firmaba lo que le presentaban y que desconocía el fraude que se estaba cometiendo, cuando claramente era su inductor. Como dicen los autores citados, un círculo vicioso difícilmente subsanable. En EE.UU., desde la ley Sarbanes-Oxley, se exige responsabilidad al máximo responsable de la preparación de la información. Con independencia del marco legal, que no deja de ser la institucionalización, el acuerdo social, de que determinadas prácticas son inaceptables o determinadas personas son responsables, existen otro instrumentos para la formación de entornos íntegros que deben ser revisados, encontrándose entre ellos la educación.

Citando a la Association of Advanced Collegiate Schools of Business (AACBS), Gonzalo y Garvey (2007) apelan al "coraje moral" como uno de los valores a desarrollar en la formación de directivos y de preparadores de la información financiera. El aprendizaje de la ética debe entrenar al alumno a decir "no" en

${ }^{5}$ EY es la nueva denominación de una de las firmas "Big Four", Ernst \& Young. 
el momento oportuno. Esta tarea no es sencilla como indica Kennedy (2006). El aprendizaje en un aula no es entrenamiento; el coraje no lo genera la situación, el coraje está en la persona y se va formando progresivamente a partir de experiencias que van requiriendo incrementalmente este coraje. Paralelamente al coraje otro valor que ayuda a mantener un tono íntegro es la disciplina, mientras que el coraje pone de manifiesto la capacidad para resistir las presiones externas a un comportamiento no ético, la disciplina ayuda a vencer las presiones internas, como los deseos y las tentaciones. El coraje libera del miedo, mientras que la disciplina lo hace de las pasiones internas. Las pasiones son positivas, son "pasiones correctas", si están destinadas a la consecución de los fines de la entidad; el riesgo del que alerta Kennedy (2006), y que lo sitúa en la base de algunos escándalos recientes, es no saber distinguir las pasiones personales de las "pasiones correctas" de la entidad. Qué duda cabe que el riesgo de fraude disminuye si desde el consejo de administración se promueven ambientes organizativos que faciliten estas virtudes personales en sus empleados.

Además del coraje moral y la disciplina, el espíritu crítico o escepticismo es un atributo deseable en los empleados, pero absolutamente necesario en las personas que asuman responsabilidades de control o supervisión (CAQ, 2010). Los indicios deben ser analizados hasta comprender las causas que los generan. El escepticismo debe reconocer la incertidumbre existente cuando se toman decisiones y que en un futuro puede poner de manifiesto que la opción elegida no resultó la mejor dado que los elementos inciertos se comportaron de manera diferente. El escepticismo es una cualidad necesaria para ejercer con responsabilidad las decisiones de supervisión o aprobación de medidas propuestas por otros. Por este motivo, el CAQ (2010) propone que esta política esté presente en los órganos de gobierno. Es más, los órganos de gobierno no son equipos (Krasna, 2006), debe existir una química especial entre los miembros del consejo, un tono de respeto y confianza en la búsqueda del bien de la entidad, especialmente porque manejan información imperfecta, pero deben mantener a salvo su independencia; el reto consiste en manejar la línea que diferencia la deslealtad y el disentimiento.

\section{Conclusiones}

La información es fundamental en el sistema financiero y para los mercados de capitales. Sobre la base de la información se toman las decisiones de asignación de recursos y se evalúa el desempeño de los directivos. La confianza en la información es una pieza clave de la arquitectura del sistema financiero. Esta confianza 
cada cierto tiempo se ve sacudida por incidentes que alertan ante posible fallos generalizados del modelo; siendo un juicio equilibrado el que debe ponderar si estamos en presencia de hechos aislados que deben servir de enseñanza o fallos estructurales del modelo que pueden dar al traste con la relación de confianza.

La información es preparada por un agente diferente al que toma las decisiones con base en la misma. Unas veces serán los propietarios al evaluar el desempeño, otras los inversores o acreedores, al decidir si asignan financiación a la entidad. Como existe un obvio conflicto de intereses en quien prepara la información, se establecen mecanismos de control de la calidad de esa información. En ocasiones, esos mecanismos funcionan en el día a día, como los mecanismos de gobierno corporativo, en otras, de manera periódica como la auditoría externa.

Los mecanismos de gobierno corporativo tratan de establecer una cultura organizativa que impida la comisión de fraudes en la información. Estos programas se sustentan en la metodología del triángulo del fraude desarrollado por Cressey. Esta metodología indica que el fraude para ser cometido requiere el concurso de un incentivo o presión, una oportunidad de cometerlo y esconderlo y una racionalización que justifique personalmente al defraudador. Las medidas para evitar el fraude se centran en esas tres dimensiones que hemos ido desarrollando a lo largo de este trabajo. La reducción del riesgo del fraude supone aislar los episodios de fraude como enseñanzas que ayudan a minimizar los incidentes futuros, pero aplicando una dosis de escepticismo sobre las verdaderas causas de esos fraudes.

Las dimensiones a trabajar requieren reformas institucionales, en nuestra opinión las menos, y mejoras en los mecanismos que garanticen la vigencia efectiva de las instituciones. Así, los modelos de gobierno corporativo se han desarrollado ampliamente en los últimos años, evolucionando desde propuestas voluntarias a medidas obligatorias, recogidas en las legislaciones como la española. Sin embargo, el fraude está estrechamente relacionado con el nivel de integridad de las personas que trabajan en las organizaciones y su vigencia efectiva requiere mejoras en los programas de entrenamiento de los directivos, así como mejoras en las culturas organizativas.

En definitiva, es un tema que preocupa, tanto al nivel de entidades privadas, como en el sector público. El fraude es un tema de investigación multidisciplinar, dándose cita disciplinas como el derecho, la psicología, la criminología y la contabilidad. En este último campo, una buena muestra del interés es que la prestigiosa American Accounting Association, recientemente le ha dedicado un espacio en su reunión anual, apuntando interesantes áreas de trabajo (Brody et al, 2012). 


\section{Bibliografía}

AlBRETCH, S., HOWE, K. y RONNEY, M. (1984): Deterring Fraud: the internal auditor's perspective. Ed. Institute of Internal Auditors research Foundation, 1-42.

Beasley, M. S., Carcello, J. V., Hermanson, D. R. y Neal, T. L. (2010): Fraudulent Financial Reporting: 1998-2007. Ed. COSO. Disponible en: hitp://www.coso. org/documents/cosofraudstudy2010 001.pdf

Boatright, J. R. (2006): "Ethics for a Post-Enron America", en Enron and World Finance. A Case Study in Ethics, ed. Dembinski, P. H. et al. Palgrave MacMillan, Newton York.

Brody, R. G., Melendy, S. R. y PerRI, F. S. (2012): "Commentary from the American Accounting Association's 2011 Annual Meeting Panel on Emerging Issues in Fraud Research", Accounting Horizons, 26(3), 513-531.

CAÑIBANO, L. y PedRosa, F. (2009): "La actuación de los supervisores ante el incumplimiento: Registros de la CMVM y CNMV". XV Congreso de AECA. Disponible en http://www.aecal.org/pub/on line/comunicaciones xvcongresoaeca/cd/75a. pdf.

Center for Audit Qualty (CAQ), (2010): Deterring and Detecting Financial Reporting Fraud. A Platform for Action. Disponible en: http://www. google.es/url? sa=t\& $r c t=i \& q=\&$ esrc $=s \& f r m=1$ \& source $=$ web\& $c d=1$ \&ved $=0 C$ CgQFjAA\&url=http\%3A\%2F\%2Fwww.thecaq.org\%2Fdocs\%2Freports-andpublications $\% 2$ Fdeterring-and-detecting-financial-reporting-fraud-a-platformfor-action.pdf\%3Fsfvrsn\%3D0\&ei=1Yv3U- H4TlaN37gfgG\&usg=AFQjCNE6P W2NyaypzAVDyUk_MB5bET6iPg\&sig2=LOMSdQqHo9vPftBB6F7uWg\&bvm=bv .73612305,d.d2s.

Comisión Nacional del Mercado de Valores (2013): Circular 5/2013, de 12 de junio, de la Comisión Nacional del Mercado de Valores, que establece los modelos de informe anual de gobierno corporativo de las sociedades anónimas cotizadas, de las cajas de ahorros y de otras entidades que emitan valores admitidos a negociación en mercados oficiales de valores. BOE 150 de 24/06/2013.

Congreso de los Diputados X Legislatura (2014): Proyecto de Ley por el que se modifica la Ley de Sociedades de Capital para la mejora del gobierno corporativo. Boletín Oficial de las Cortes Generales 97-1. 
DIARIO EXPANSIÓN (2014): Bankia: CNMV y Banco de España discrepan con el ICAC por Deloitte. 17/07/2014. Disponible en: http://www.expansion.com/2014/07/17/ empresas/banca/1405623317.html

DoBSON, J. (2006): "Enron The Collapse of Corporate Culture" en Enron and World Finance. A Case Study in Ethics, ed. Dembinski, P. H. et al. Palgrave MacMillan, Newton York.

EY (2014): Overcoming compliance fatigue. Reinforcing the commitment to ethical growth. Disponible en: http://www.ey.com/Publication/vwLUAssets/Overcoming compliance fatigue/\$FILE/13th\%20GLOBAL\%20FRAUD\%20SURVEY\%20 FINAL\%20low\%20res.pdf

Erickson, M., Hanlon, M. y MAYdeW, E. (2004): "Is there a Link Between Executive Compensation and Accounting Fraud" Working paper. Disponible en: http:// leeds-faculty.colorado.edu/bhagat/execcompacctfraud.pdf.

Gonzalo, J. A. y GarveY, A. (2007): "Ética y enseñanza de la contabilidad: una propuesta de discusión". Contaduría Universidad de Antioquia, 50, 11-42.

GRUPO de ACCIÓN FINANCIERA INTERNACIONAL (GAFI) (2012): Estándares Internacionales sobre la Lucha contra el Lavado de Activos y el Financiamiento del Terrorismo y la Proliferación. Las Recomendaciones del GAFI. Disponible en: http://www.google.es/ urle sa $=t \& r c t=i \& q=\&$ esrc $=s \& f r m=1$ \& source=web\&cd=2\&ved=0CCsQFjAB\&url=http \%3A\%2F\%2Fwww.gafisud.info\%2Fdocumentos\%2Fesp\%2FLas Nuevas 40 Recomendaciones.pdf\&ei=kZX5U7n-LPDZOQW6pIDgBg\&usg=AFQjCNFixJzSwkRwiwH $x 5$ YmObCjudxFafw\&sig2=qL6FI -yrs1 bEVyqB3uAQ\&bvm=bv.73612305,d.d2k.

International Federation of Accountants (2009): International Standard on Auditing 240: The auditor's responsibilities relating to fraud in an audit of financial statements. Disponible en http://www.ifac.org/sites/default/files/downloads/ a012-2010-iaasb-handbook-isa-240.pdf.

JeNNINGS, M. M. y HAPPEL, S. (2006): "The Post-Enron Era for Stakeholder Theory: A New Look at Corporate Governance and the Coase Theorem" en Enron and World Finance. A Case Study in Ethics, ed. Dembinski, P. H. et al. Palgrave MacMillan, Newton York.

KASSEM, R. y HIGSON, A. (2012): "The New Fraud Triangle Model", Journal of Emerging Trends in Economics and Management Series, 3(3), 191-195. 
KENNEDY, R.G. (2006): "Ethics, Courage and Discipline: The Lessons of Enron", en Enron and World Finance. A Case Study in Ethics, ed. Dembinski, P. H. et al. Palgrave MacMillan, Newton York.

Krasna, B. (2006): "Enron Revisited: What Is a Board Member to Do?", en Enron and World Finance. A Case Study in Ethics, ed Dembinski, P. H. et al. Palgrave MacMillan, Newton York.

Mesmer-Magnus, J.y Viswesvaran, C. (2005): "Whistleblowing in Organizations: An Examination of Correlates of Whistleblowing Intentions, Actions, and Retaliation". Journal of Business Ethics 62, 277-297.

Sauviat, C. (2006): "The Demise of Andersen" en Enron and World Finance. A Case Study in Ethics, ed Dembinski, P. H. et al. Palgrave MacMillan, Newton York.

Vadera, A. K., Aguilera, R. V. y CazA, B. B. (2009): "Making Sense of WhistleBlowing's Antecedents: Learning from Research on Identity and Ethics Programs". Business Ethics Quarterly 19(4), 553-586.

Wolfe, D.T. y HERMANSON, D. R. (2004): "The fraud diamond: considering the four elements of fraud", The CPA Journal, December, 1-5.

ZefF, S. A. (2003): "How the U.S. accounting profession got where it is today: part II". Accounting Horizons, 7(4), 267-286.

- (1995): Ley 2/1995, de 23 de marzo, de Sociedades de Responsabilidad Limitada. BOE 71 de 24/03/1995.

- (2002): Ley 44/2002, de 22 de noviembre, de Medidas de Reforma del Sistema Financiero. BOE 281, 23/11/2002.'

- (2010): Ley 10/2010, de 28 de abril, de prevención del blanqueo de capitales y de la financiación del terrorismo. BOE 103 de 29/04/2010.

- (2011): Ley 2/2011, de 4 de marzo, de Economía Sostenible. BOE 55 de $5 / 03 / 2011$.

- (2014): Directiva 2014/56/UE, de 16 de abril de 2014, por la que se modifica la Directiva 2006/43/CE relativa a la auditoría legal de las cuentas anuales y de las cuentas consolidadas. DOCE 27/05/2014. 
- (2014): Reglamento 537/2014, de 16 de abril, sobre los requisitos específicos para la auditoría legal de las entidades de interés público y por el que se deroga la Decisión 2005/909/CE de la Comisión. DOCE 27/05/2014.

- (2014): Real Decreto 304/2014, de 5 de mayo, por el que se aprueba el Reglamento de la Ley 10/2010, de 28 de abril, de prevención del blanqueo de capitales y de la financiación del terrorismo. BOE 110 de 6/05/2014.

- (2014): Ley 31/2014, de 3 de diciembre, por la que se modifica la Ley de Sociedades de Capital para la mejora del gobierno corporativo. BOE 293 de 4 de diciembre de 2014. Disponible en: http://www.boe.es/boe/dias/2014/12/04/ pdfs/BOE-A-2014-12589.pdf (Consultado 22 de febrero de 2015). 